\title{
FLORA DA BAHIA: LEGUMINOSAE - ANCISTROTROPIS (PAPILIONOIDEAE) E FILOGENIA DO GÊNERO ANCISTROTROPIS
}

\author{
Felipe da Silva Santos'; Luciano Paganucci de Queiroz²; Cristiane Snak³
}

\author{
1. Bolsista PROBIC, Graduando em Bacharelado em Ciências Biológicas, Universidade Estadual de Feira de Santana, email: \\ santos.felipeuefs@gmail.com \\ 2. Orientador, Departamento de Ciências Biológicas, Universidade Estadual de Feira de Santana, email: \\ luciano.paganucci@gmail.com \\ 3. Coorientadora, Departamento de Ciências Biológicas, Universidade Estadual de Feira de Santana, email: \\ cristianesnak@gmail.com
}

PALAVRAS-CHAVE: Phaseolineae, taxonomia, Vigna s.l.

\section{INTRODUÇÃO}

Leguminosae compreende cerca de 19.500 espécies e 770 gêneros, dos quais 220 gêneros e cerca de 2.835 espécies ocorrem no Brasil. A Bahia, com cerca de 937 espécies, é o segundo estado com maior diversidade (BFG 2015, LPWG 2013, 2017). A família é dividida em seis subfamílias: Cercidoideae, Detarioideae, Dialioideae, Duparquetioideae, Caesalpinioideae e Papilionoideae, sendo as cinco primeiras segregadas de Caesalpinioideae s.l. e Mimosoideae incluída em Caesalpinioideae (LPWG 2017). A circunscrição de Papilionoideae não teve alterações e ela inclui 29 tribos, 503 gêneros e cerca de 14.000 espécies (LPWG 2013, 2017, Queiroz et al. 2015).

Phaseoleae é a tribo mais diversa, tendo como características principais hábito volúvel e as folhas trifoliadas (Bruneau et al. 1995), e dentre suas diversas subtribos, Phaseolineae é a que possui o maior número de espécies. Phaseolineae é um grupo monofilético (Kajita et al. 2001) mas, apesar disso, possui diversos problemas de delimitação entre os gêneros e entre as espécies de diversos gêneros. Dentre esses gêneros, Phaseolus e Vigna formam um complexo que há muito tempo vem sendo estudado pela sua dificuldade de delimitação (Maréchal et al. 1978). Em 2011 esse complexo foi estudado por Delgado-Salinas et al., que segregou o gênero Vigna Savi em sete gêneros: Ancistrotropis A. Delgado, Cochliasanthus Trew, Condylostylis Piper, Helicotropis A. Delgado, Leptospron (Benth) A. Delgado, Sigmoidotropis (Piper) A. Delgado, e Vigna (s.s.).

\section{METODOLOGIA}

Este trabalho teve como área de estudo o estado da Bahia, Brasil, no qual foram realizadas duas viagens para coleta de amostras e foram revisados os espécimes do gênero nos herbários ALCB, CEPEC, HUEFS e HURB, RB [acrônimos de acordo com Thiers (2017)].

Para este trabalho foram examinadas cerca de 150 exsicatas. A identificação das espécies de Ancistrotropis foi feita utilizando as obras príncipes e os tipos nomenclaturais das espécies da Bahia e Brasil e seus sinônimos. Também foram utilizados trabalhos taxonômicos sobre o gênero. As descrições morfológicas foram feitas a partir das exsicatas. As medidas foram tomadas em estruturas adultas. A terminologia morfológica geral seguiu a proposta por Gonçalves \& Lorenzi (2011) e Radford et al. (1976), termos específicos para o gênero por Maréchal et al. (1978) e Delgado-Salinas et al. (2011).

Cada espécie foi descrita, foram feitas algumas ilustrações, além de serem apresentados dados de sua distribuição geográfica na Bahia, seguindo o modelo proposto para o projeto Flora da Bahia (http://www1.uefs.br/floradabahia/). 
Quanto à parte molecular, o estudo filogenético contém múltiplos acessos de cada espécie de Ancistrotropis a fim de testar o monofiletismo do gênero, tentar resolver os problemas de delimitação e conhecer as relações filogenéticas entre as espécies do gênero. O número de acessos por espécie tentará cobrir a variação morfológica observada em cada espécie, sua distribuição geográfica e os habitats nos quais ocorre. As amostras necessárias para o desenvolvimento deste estudo foram obtidas através de material preservado em sílica-gel coletado em campo e através de coleções de herbário, quando não foi possível obter amostras em campo. A extração de DNA, a obtenção de sequências de nrITs e trnK-matK e as análises filogenéticas de máxima parcimônia e Bayesiana foram realizadas de acordo com os procedimentos descritos em Queiroz et al. (2015).

\section{RESULTADOS E DISCUSSÃO}

Ancistrotropis é caracterizado principalmente por suas flores, com cálice campanulado, 4laciniado; estandarte formando uma estrutura que lembra um capuz, com aurículas salientes em forma de gancho; alas arredondadas mais longas que a carena; pétalas da carena sigmoides, rostro em forma de gancho, margens internas não conatas, mas fechadas por tricomas marginais entrelaçados e conspícuos; gineceu com estilete prolongado além do estigma que é lateral.

Ancistrotopis é um gênero Neotropical, possuindo ao todo sete espécies descritas (DelgadoSalinas et al. 2011). No Brasil existe o registro para seis espécies: A. arrabidae (Steud) A. Delgado, A. clitorioides (Mart. ex Benth) A. Delgado, A. firmula (Mart. ex Benth.) A. Delgado, A. peduncularis (Fawc. \& Rendle) A. Delgado, A. robusta (Piper) A. Delgado e A. serrana Snak, J.L.A. Moreira \& A.M.G. Azevedo (Delgado-Salinas et al. 2011, Snak et al. 2014). No Brasil, o gênero ocorre em todas as regiões, estando presente em seus principais domínios fitogeográficos, como Amazônia, Caatinga, Cerrado, Mata Atlântica e Pantanal (BFG 2015). Para o estado da Bahia foram registradas quatro espécies: A. clitorioides, A. firmula, A. peduncularis, A. serrana. Na Bahia, as espécies ocorrem principalmente em áreas de caatinga e cerrado, numa faixa de altitude de 400-1.400 m. Das quatro espécies que ocorrem no estado, A. firmula, A. serrana, possuem uma delimitação morfológica bem distinta sendo de fácil identificação, já A. peduncularis e $A$. clitoriodes tem limites morfológicos imprecisos, sendo difícil a diferenciação entre elas, o que explica porque essas espécies são identificadas erroneamente em muitos herbários.

Em análises de Máxima Parcimônia e Inferência Bayesiana realizadas com as sequências obtidas de Ancistrotropis as árvores resultantes recuperaram topologias semelhantes. $\mathrm{O}$ gênero Ancistrotropis foi recuperado com alto suporte (1.0 PP) como grupo irmão de Condylostyles (Piper). Este clado formado por Ancistrotropis + Condylostyles é fortemente sustentado como grupo irmão de Sigmoidotropis + gênero novo (1.0 PP). Esses relacionamentos entre Ancistrotropis, Condylostyles e Sigmoidotropis já haviam sido demonstrados no trabalho de Delgado-Salinas (2011), entretanto sem suporte estatístico significativo. Dentro de Ancistrotropis, verificou-se que $A$. firmula morfotipo crassifolius, possui um alto suporte nas duas análises, como irmã de um clado formado por A. firmula típica (1.0 PP, $100 \mathrm{BS}$ ) (Fig. 2). Um dos morfotipos de A. clitorioides aparece como irmã do clado formado por A. peduncularis (0.98 PP, $63 \mathrm{BS}$ ) (Fig. 2). Um dos morfotipos de A. peduncularis aparece como A. clitorioides (0.55 PP, 88 BS) (Fig. 2). Esses resultados preliminares indicam que ainda é complicado estabelecer um limite morfológico entre as espécies $A$. clitorioides e A. peduncularis, necessitando de uma maior amostragem para estudos. Além disso mostra a importância do estudo filogenético no gênero com auxílio morfológico, para entender a relação entre suas espécies e poder definir os limites entre elas.

Por fim, um morfotipo que corresponderia a espécie nova (Fig. 1) de Ancistrotrotropis, devido apresentar as características morfológicas diagnósticas do gênero, foi recuperada como um grupo 
irmão de Sigmoidotropis (0.99 PP, 88 BS) (Fig. 2), caracterizando então uma nova linhagem dentro de Phaseolinae.

\section{CONSIDERAÇÕES FINAIS}

Com esse trabalho é possível verificar que Ancistrotropis não é bem definido na relação entre as espécies $A$. clitorioides e A. pedunculares, sendo possível notar isso tanto na parte morfológica, quanto na parte molecular. A circunscrição atual de Ancistrtropis firmula deve ser restringida para excluir o morfotipo antes denominado Phaseolus crassifolius, que foi demonstrado como uma espécie distinta que será restabelecida.

Com o estudo filogenético foi possível também perceber, além do novo gênero e espécie, que o grupo de gêneros irmãos de Ancistrotropis possuem limites ainda imprecisos quanto a morfologia de suas espécies, dificultando o estudo dos mesmos, e que os dados moleculares são de importância para dar suporte em futuros estudos nesses grupos em conjunto.
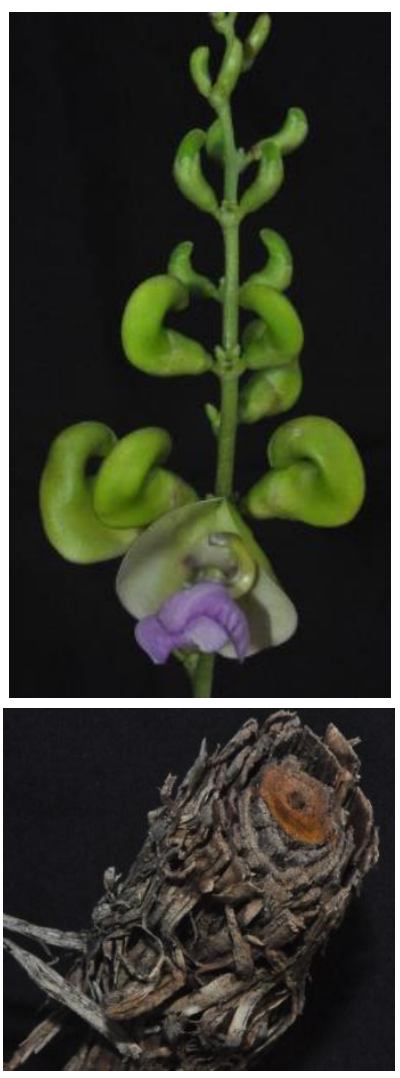

Figura 1: A: inflorescência, B: ramo. Partes do gênero novo que se destacam em comparação a Ancistrotropis.

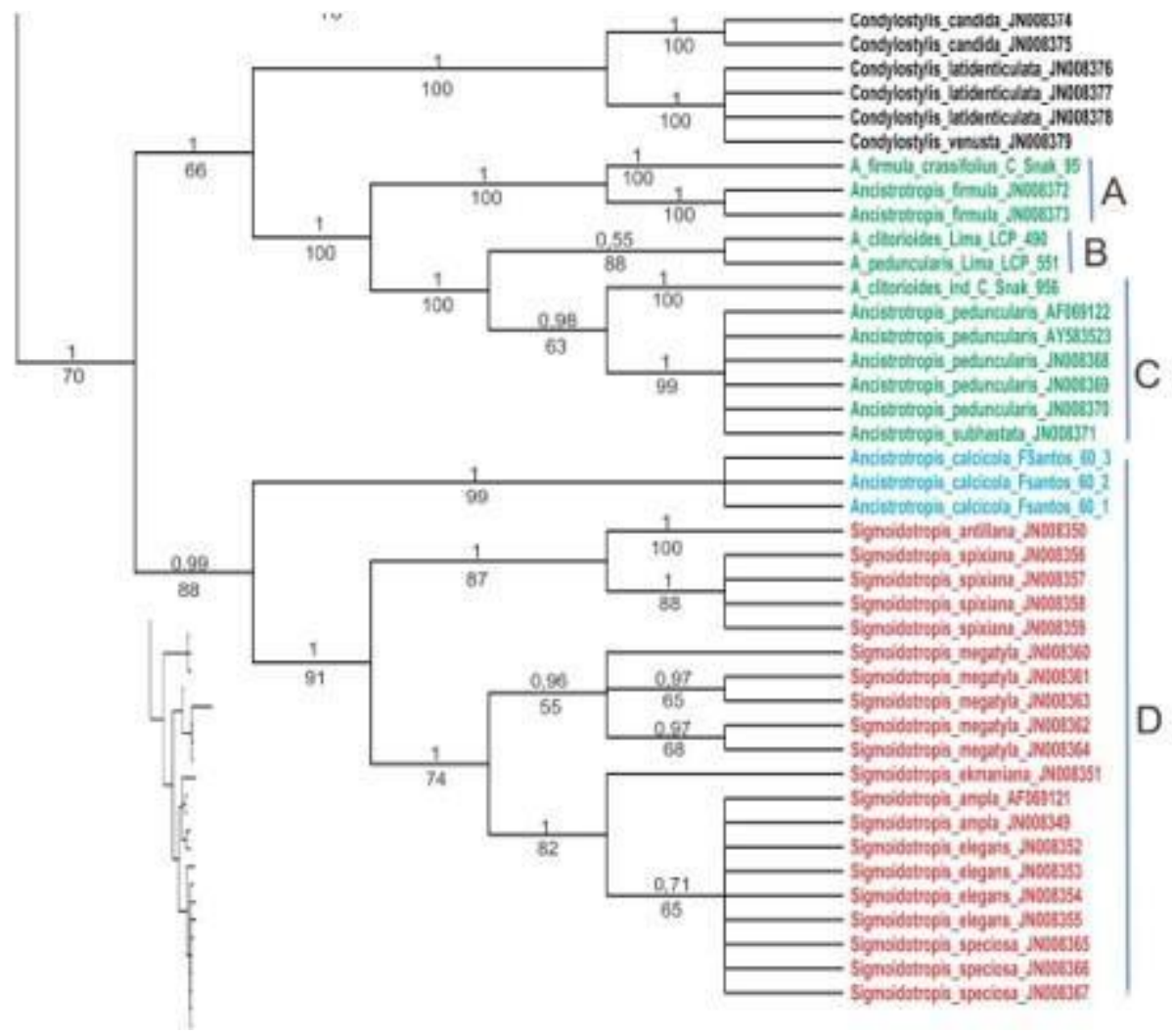

Figura 2: Árvore de consenso de maioria resultante da análise Bayesiana baseada em dados de ITS. Os valores de suporte de probabilidade posterior se encontram acima dos ramos e os valores bootstrap se encontram abaixo dos ramos. 


\section{REFERÊNCIAS}

BFG [The Brazil Flora Group] 2015. Growing knowledge: an overview of Seed Plant knowledge in Brazil. Rodriguésia 66: 1085-1113.

BRUNEAU, A., DOYLE, J.L.; DOYLE, J.J. 1995. Phylogenetic evidence in Phaseoleae: evidence from chloroplast restriction site characters. In: M.D. Crisp \& J.J. Doyle [eds.]. Advances in Legume Systematics: Phylogeny. Part 7. The Royal Botanic Gardens, Kew, p. 309-330.

DELGADO-SALINAS, A., THULIN, M., PASQUET, R., WEEDEN, N.; LAVIN, M. 2011. Vigna (Leguminosae) sensu lato: The Names and identities of the American segregate genera. Am. J. Bot. 98: 1694-1715.

GONÇALVES, E.G.; LORENZI, H. 2011. Morfologia Vegetal: Organografia e Dicionário Ilustrado de Morfologia das Plantas Vasculares. Instituto Plantarum de Estudos da Flora, 2. Ed. São Paulo.

KAJITA, T., OHASHI, H., TATEISHI, Y., BAILEY, C.D.; DOYLE J.J. 2001. Rbcl and legume phylogeny, with particular reference to Phaseoleae, Millettieae, and allies. Syst. Bot. 26: 515536.

LACKEY, J.A. 1981. Phaseolae. In: Polhill, R.M.; Raven, P.H. [eds.]. Advances in Legume Systematics, part 1. Royal Botanic Gardens, Kew, pp. 301-327.

LPWG [Legume Phylogeny Working Group]. 2013. Legume phylogeny and classification in the 21st century: Progress, prospects and lessons for other species-rich clades. Taxon 62: 217-248.

LPWG [Legume Phylogeny Working Group]. 2017. Phylogeny and classification of the Leguminosae. Taxon 66: 44-77.

MARÉCHAL, R., MASCHERPA, J.; STAINER, F. 1978. Etude taxonomique d'un groupe complexe d'espècies des genres Phaseolus et Vigna (Papilionoideae) sur la base de donées morphologiques et polliniques, traitées par l'analyse informatique. Boissiera 28: 1-273.

QUEIROZ, L.P., PASTORE, J.F.B., CARDOSO, D., SNAK, C., LIMA, A.L.C., GAGNON, E., VATANPARAST, M., HOLLAND, A.E.,; EGAN, A.E. 2015. A multilocus phylogenetic analysis reveals the monophyly of a recircumscribed papilionoid legume tribo Diocleae with well-supported generic relationships. Mol. Phylogenet. Evol. 90: 1-19.

RADFORD, A.E., DICKISON, W.C., MASSEY, J.R.; BELL, C.R. 1976. Vascular Plant Systematics. Harper and Row, New York.

SNAK, C., MOREIRA, J.L.A.; TOZZI, A.M.G.A. 2014. A new species of Ancistrotropis (Leguminosae, Papilionoideae, Phaseoleae) endemic to Brazil. Phytotaxa 172: 280-284.

THIERS, B. [atualizado continuamente]. Index Herbariorum: A global directory of public herbaria and associated staff. New York Botanical Garden's Virtual Herbarium. Available from: http://sweetgum.nybg.org/ih/ (acesso: 03 de agosto de 2017). 